Kalaycıoğlu Ezgi ${ }^{1}$, Çetin Mustafa ${ }^{2}$, Özyıldız Ali Gökhan ${ }^{2}$, Kırış Tuncay ${ }^{3}$, Turan Turhan ${ }^{1}$

${ }^{1}$ University of Health Sciences Ahi Evren Chest and Cardiovascular Surgery

Training and Research Hospital, Cardiology Department, Trabzon, Turkey

${ }^{2}$ Recep Tayyip Erdoğan University Training and Research Hospital, Cardiology Department, Rize Turkey

${ }^{3}$ Katip Çelebi University Atatürk Training and Research Hospital, Cardiology Department, İzmir, Turkey

\title{
Is Adiponectin Elevation Associated WiTh LEFT ATRIAL REMODELING AND IMPAIRED Mechanical Functions? (a Speckle Tracking Study)
}

\begin{abstract}
Objectives
Material and methods

Results

Conclusion

Keywords

For citation

Recent studies demonstrated that elevated adiponectin levels predicted an increased risk of atrial fibrillation (AF) and stroke; however, a causal relationship is yet to be unknown. Reduced left atrium (LA) functions detected by two-dimensional echocardiographic speckle tracking (2D-STE) can predict $\mathrm{AF}$ development. We aimed to investigate the relationship between adiponectin level and LA functions in hypertensive and diabetic patients at high risk for incident AF.

The study consisted of 80 hypertensive diabetic patients. All patients underwent echocardiography, and venous blood samples were taken. The relationship between adiponectin levels and LA functions was analyzed.

We divided patients into two groups according to the mean adiponectin level $(13.63 \mathrm{ng} / \mathrm{ml})$. In the high adiponectin group, the mean age $(\mathrm{p}=0.001)$ and high-density lipoprotein (HDL) cholesterol $(\mathrm{p}=0.015)$ were higher, whereas estimated glomerular filtration rate (eGFR) $(\mathrm{p}=0.036)$ and hemoglobin $(\mathrm{p}=0.014)$ levels were lower. Although LA maximum volume, LA minimum volume, and LA pre-A volume were higher in the group with high adiponectin levels, they did not reach a statistical significance. Peak early diastolic LA strain $(\mathrm{S}-\mathrm{LAe})(\mathrm{p}=0.048)$ and strain rate (SR-LAe) $(\mathrm{p}=0.017)$ were lower in this group. Multivariate logistic regression analysis demonstrated that age $(\mathrm{p}=0.003)$ and hemoglobin $(\mathrm{p}=0.006)$ were predictors of elevated adiponectin levels. On the contrary, $\mathrm{S}$-LAe, HDL cholesterol, and eGFR lost their statistical significance.

In patients with $\mathrm{HT}$ and DM, elevated adiponectin level is associated with impaired LA mechanical functions. Increased age and hemoglobin level are independent predictors of elevated adiponectin levels.

Adiponectin; atrial mechanical functions; 2D- speckle tracking echocardiography

Kalaycıŏlu Ezgi, Çetin Mustafa, Özyıldız Ali Gökhan, Kırış Tuncay, Turan Turhan. Is Adiponectin Elevation Associated with Left Atrial Remodeling and Impaired Mechanical Functions? (a Speckle Tracking Study). Kardiologiia. 2021;61(5):65-70. [Russian: Камайсоглу Езги, Четин Мустафа, Озыымдыз Али Гейкан, Кириш Тункей, Туран Турхан. Связано ми повышение уровня адипонектина с ремоделированием мевого преАсердия и нарушением его механической функции? (с использованием технологии Speckle Tracking). Кардиология. 2021;61(5):65-70]
\end{abstract}

Corresponding author

Ali Gökhan Özyıldız. E-mail: aligokhanozyildiz@gmail.com

\section{Introduction}

Adiponectin is a peptide hormone with insulin-sensitizing, anti-inflammatory, and anti-atherosclerotic effects, and it is presumed to be protective against cardiovascular (CV) diseases [1]. However, elevated adiponectin concentration is associated with adverse $\mathrm{CV}$ outcomes, including coronary heart disease, heart failure, and allcause mortality. Vascular injury caused by atherosclerosis results in elevated adiponectin, which is considered the main link between adiponectin and adverse outcomes [2-4]. Recent studies demonstrated that elevated adiponectin predicted an increased risk of atrial fibrillation (AF) and stroke. However, a causal relationship is yet to be established $[5,6]$.
Left atrial (LA) remodeling involves several pathologic processes, including atrial fibrosis, electrical remodeling, and loss of atrial myocytes [7]. Recent evidence suggests that LA fibrosis is the leading prerequisite for initiation, progression, and persistence of AF. In clinical practice, remodeling is determined by echocardiographic LA enlargement and dysfunction rather than by detection of LA fibrosis.

Unlike conventional echocardiography, evaluation of the LA with two-dimensional echocardiographic speckle tracking (2D-STE) may evaluate reduced LA functions in the subclinical stage [8]. Additionally, reduced LA functions detected by 2D-STE can predict AF development [9]. 
Hypertension (HT) and diabetes mellitus (DM) are primary triggers causing LA remodeling and subsequent AF. Obesity and a sedentary lifestyle contribute to the incidence and coexistence of these two diseases [10]. In the present study, we hypothesized that adiponectin had contributed to LA remodeling. Hence, we aimed to investigate the relationship between adiponectin concentration and other conventional LA functional variables with LA speckle tracking in hypertensive and diabetic patients who are considered at high risk for incident AF.

\section{Material and methods \\ Study population}

The study was a single-center, cross-sectional research that initially included 100 consecutive patients who received anti-diabetic and anti-hypertensive medical treatment for at least one year. Exclusion criteria were coronary heart disease, left ventricular ejection fraction $<50 \%$, chronic renal failure, $\mathrm{AF} /$ atrial flutter, history of cerebrovascular disease, second or third-degree atrioventricular block, thyroid dysfunction, and moderate to severe valvular heart disease. Twenty patients with poor echocardiographic windows were excluded, and we analyzed the remaining 80 patients.

Hyperlipidemia was defined as statin use or low-density lipoprotein (LDL) $>70 \mathrm{mg} / \mathrm{dl}$. Body-mass index (BMI) was calculated by the formula: weight $(\mathrm{kg}) /$ height $\left(\mathrm{m}^{2}\right)$. Patients gave informed consent, and the local ethics board approved the study.

\section{Laboratory measurements}

Blood samples for determination of adiponectin were collected in EDTA tubes and centrifuged, and the isolated plasma was stored at $-80^{\circ} \mathrm{C}$ until analysis. Plasma adiponectin was measured by a sandwich ELISA assay using commercially available antibodies (Biovendor, Czech Republic).

Peripheral venous blood samples were drawn after overnight fasting and analyzed using vacuum tubes containing EDTA for storage. Hemoglobin, hemoglobin A1c, C-reactive protein (CRP), fasting glucose, total and highdensity lipoprotein (HDL) cholesterol concentrations were measured. Concentrations of low-density lipoprotein were calculated using the Friedewald equation. The estimated glomerular filtration rate (eGFR) was calculated by the Modification of Diet in Renal Disease (MDRD) Study equation [11].

\section{Conventional Echocardiography}

The echocardiographic studies were performed using an echocardiography device (VIVID S-5 General Electric Medical System Vingmed Ultrasound AS, Horten, Norway, with software for speckle-tracking of the LV) equipped with a $3.6-\mathrm{MHz}$ transducer. Measurements were obtained by a cardiologist, blinded to results of adiponectin values, according to the American Society of Echocardiography guidelines [12].

LA volume was calculated by the apical four-chamber (A1) and apical two-chamber (A2) measurements using the area-length $(\mathrm{L})$ method $(0.85(\mathrm{~A} 1 \times \mathrm{A} 2))$. The volume measurements were grouped as 1) LA maximum volume (at ventricular end-systole, just before mitral valve opens), 2) LA minimum volume (at ventricular enddiastole, when the mitral valve closed), and 3) LA pre-A volume (immediately before atrial systole, prior to the electrocardiographic $\mathrm{P}$ wave).

Formulas:

1) LA Emptying Volume $=$

(LA maximum volume - LA minimum volume);

2) LA Emptying Fraction (\%) = (LA maximum volume -

LA minimum volume)/LA maximum volume;

3) LA Active $\mathrm{EF}(\%)=[$ (LA pre-A volume -

LA minimum volume)/LA pre-A volume];

4) LA Passive $E F(\%)=[$ (LA maximum volume LA pre-A volume)/LA maximum volume];

5) LA Expansion index $=$ [ (LA maximum volume -

LA minimum volume)/LA minimum volume].

\section{Two-dimensional Speckle Tracking Analysis}

For analyzing the LA strain, two-dimensional gray-scale images of the LA were acquired in two- and four-chamber views at end-expiratory apnea during five cardiac cycles. Using 2D strain software (EchoPAC108.1.12, General Electric Medical Systems, Horten, Norway), the LA endocardial surface was manually traced using a point and click approach, which permitted automatic definition of an interested region. The software divided the region into six segments and scored the tracking quality for each segment as acceptable or non-acceptable. The non-acceptable segments were excluded from the analysis. Then, global LA strain and strain rate variables were assessed as the average of six segments. The values of LA function variables including peak early diastolic LA strain and strain rate (S-LAe and SR-LAe), peak LA strain and strain rate during ventricular systole (S-LAs and SR-LAs), and peak LA strain and strain rate during atrial systole (S-LAa and SR-LAa) were obtained from mean values of the apical two- and fourchamber views (Figure 1).

\section{Reproducibility}

For calculation of intra-observer variability of the LA strain measurements, the evaluation was repeated in 20 randomly selected patients after one month by the same investigator. For calculation of inter-observer variability, 
Figure 1. Two-dimensional speckle tracking imaging in the apical two-chamber view

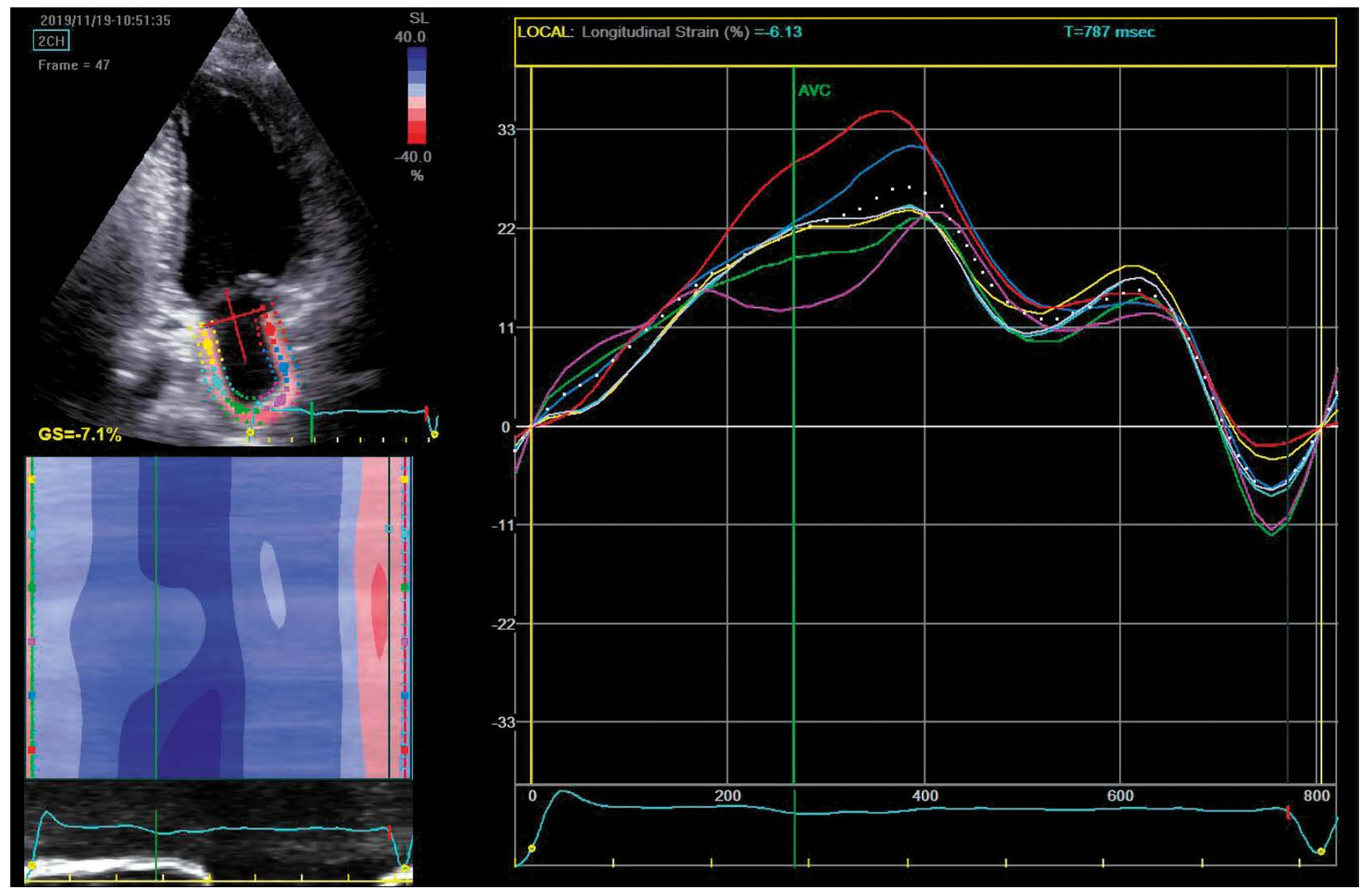

the evaluation was repeated in the same 20 patients by a different investigator, unaware of the initial study results. Intra- and inter-observer variabilities were calculated as the absolute difference between the corresponding two measurements expressed as their mean percentage. The intra-observer coefficient of variation for measurements of S-LAs, S-LAe, and S-LAa were 2.3\%, 2.2\%, and 1.9\%, respectively. The interobserver coefficient of variation for measurement of S-LAs, S-LAe, and S-LAa were 2.7\%, 2.1\%, and $3.1 \%$, respectively.

\section{Statistical Analysis}

Continuous variables are presented as mean values \pm standard deviation (SD) or as medians with ranges. Categorical variables are expressed as percentages. Normally distributed, continuous variables were compared with a two-tailed Student t-test or with an ANOVA. Nonnormally distributed variables were compared with a Mann-Whitney $U$ test or with a Kruskal-Wallis test. A chi-square test was used to analyze categorical variables. The effects of various variables on OPG were calculated by univariate logistic regression analysis. Logistic regression analyses were used for multivariate analysis of independent variables that were included if they were significantly different in the univariate analyses. Inter-observer agreement of echocardiographic variables obtained from 2D-STE data was calculated using Bland-Altman analysis, and the intra-class correlation coefficient was used to assess intra-observer agreement. All the statistical tests were twotailed, and a $\mathrm{p}<0.05$ value was considered significant. All the analyses were carried out using SPSS version 15 (SPSS, Inc., Chicago, Illinois, US).

\section{Results}

The study included 80 consecutive patients ( 46 female) with a mean age of 58.3 years. According to the adiponectin's mean value $(13.63 \mathrm{ng} / \mathrm{ml})$, the patients were divided into two groups. The laboratory and demographic data of the groups were compared with univariate analysis. In the group with high adiponectin, the mean age $(\mathrm{p}=0.001)$ and high-density lipoprotein (HDL) cholesterol $(\mathrm{p}=0.015)$ were higher, whereas eGFR $(\mathrm{p}=0.036)$ and hemoglobin $(\mathrm{p}=0.014)$ were lower (Table 1). Although it did not reach statistical significance, office systolic and diastolic blood pressure, hemoglobin Alc, and fasting glucose tended to be higher in this group.

Echocardiographic values are shown in Table 1. In conventional echocardiography, there was no difference between LA emptying volume, LA emptying fraction, LA active-passive ejection fraction (EF), and LA expansion index 
Table 1. Clinical demographic, laboratory, and echocardiographic characteristics of the groups

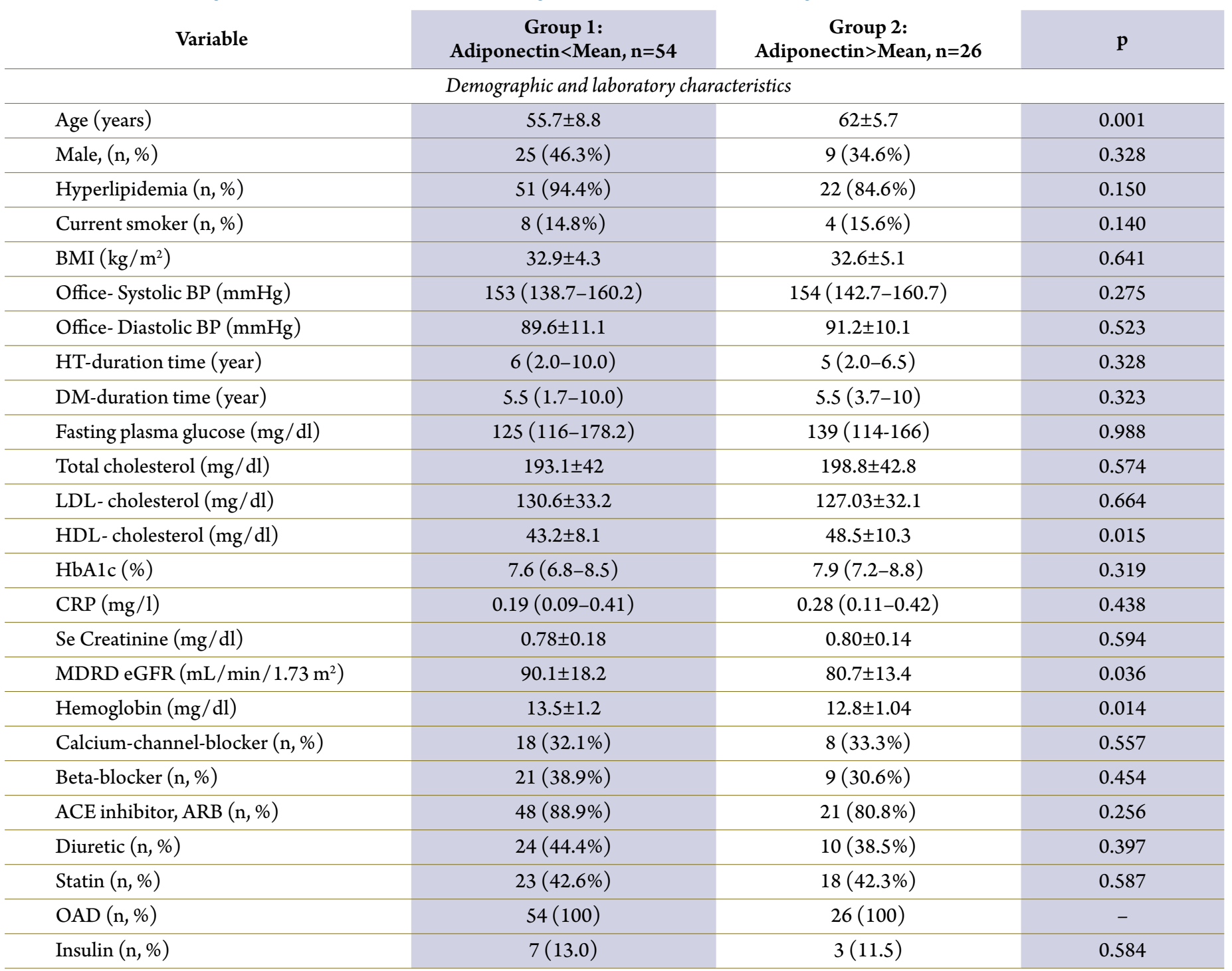

Echocardiographic Variables

\begin{tabular}{|c|c|c|c|}
\hline LA MinVol (ml) & $23.73(18.6-30.7)$ & $27.35(18.7-35.5)$ & 0.116 \\
\hline LA MaxVol (ml) & $50.4(40.8-62.3)$ & $52.9(41.4-66.7)$ & 0.302 \\
\hline LA Pre A Vol (ml) & $34.6(30.3-47.8)$ & $39.1(29.5-52.3)$ & 0.187 \\
\hline LA Empty Frak \% & $52.7 \pm 10.4$ & $48.9 \pm 10.1$ & 0.129 \\
\hline LA Pasif EF \% & $25.9 \pm 11.2$ & $27.6 \pm 8.5$ & 0.530 \\
\hline LA ExpInd & $1.05(0.89-1.4)$ & $1.06(0.78-1.34)$ & 0.213 \\
\hline S-LAs (\%) & $36.3 \pm 9.2$ & $32.7 \pm 7.8$ & 0.091 \\
\hline S-LAe (\%) & $18.02 \pm 6.2$ & $15.2 \pm 4.5$ & 0.048 \\
\hline S-LAa (\%) & $16.8(15.3-21.9)$ & $17.8(12.8-20.8)$ & 0.549 \\
\hline SR-LAs (s-1) & $1.52 \pm 0.43$ & $1.36 \pm 0.31$ & 0.077 \\
\hline
\end{tabular}

Data are mean \pm SD or median and (range, [25\% percentile-75\% percentile]). BMI, body mass index; BP, blood pressure; HT, hypertension; DM, diabetes mellitus; LDL, low-density lipoprotein; HDL, high density lipoprotein; CRP, C-reactive protein; MDRD, modification of diet in renal disease; eGFR, estimated glomerular filtration fraction; ACE, angiotensin converting enzyme; $\mathrm{ARB}$, angiotensin receptor blocker; OAD, oral anti-diabetic; LA, left atrium; S-LAs, peak left atrial strain during ventricular systole; S-LAe, peak left atrial strain at early diastole; S-LAa, peak left atrial strain at atrial systole; SR-LAs, peak left atrial strain rate during ventricular systole; SR-LAe, peak left atrial strain rate at early diastole; SR-LAa, peak left atrial strain rate at atrial systole. 
Table 2. Logistic regression analysis of the relationship between variables and adiponectin

\begin{tabular}{|c|c|c|c|c|c|c|}
\hline \multirow{2}{*}{ Variable } & \multicolumn{3}{|c|}{ Univariate analysis } & \multicolumn{3}{|c|}{ Multivariate analysis } \\
\hline & OR & 95 CI\% & $\mathbf{p}$ & OR & 95 CI\% & $\mathbf{p}$ \\
\hline Age & 1.108 & $1.036-1.185$ & 0.003 & 1.149 & $1.047-1.260$ & 0.003 \\
\hline HDL & 1.067 & $1.010-1.126$ & 0.020 & 1.070 & $0.990-1.156$ & 0.088 \\
\hline $\mathrm{Hgb}$ & 0.563 & $0.350-0.906$ & 0.018 & 0.394 & $0.178-0.874$ & 0.006 \\
\hline GLSRe LA & 0.171 & $0.041-0.703$ & 0.014 & - & - & - \\
\hline
\end{tabular}

MDRD, Modification of Diet in Renal Disease; eGFR, Estimated glomerular filtration fraction;

S-LAe, peak left atrial strain at early diastole; SR-LAe, peak left atrial strain rate at early diastole.

measurements that reflected LA systolic, diastolic and booster pump functions. Although the values of LA maximum volume, LA minimum volume, and LA pre-A volume were higher in the group with high adiponectin, they did not reach statistical significance. In LA strain and strain rate analyses, S-LAe $(\%)(\mathrm{p}=0.048)$ and SR-LAe $(\mathrm{s}-1)(\mathrm{p}=0.017)$ were lower in the group with high adiponectin. S-LAs (\%) tended to be was lower in this group but not statistically significant $(\mathrm{p}=0.091)$. There were no differences in other variable between the groups (Table 1).

Parameters found to be significant in the univariate analysis were then included in a backward multivariate logistic regression analysis. The results showed that age (OR $1.149,95 \%$ CI 1.047-1.260, $\mathrm{p}=0.003$ ) and hemoglobin (OR $0.394,95 \%$ CI $0.178-0.874, p=0.006)$ were predictors of elevated adiponectin. Conversely, S-LAe, HDL cholesterol, and eGFR lost statistical significance (Table 2).

\section{Discussion}

In the current study, we found a decrease in LA functions as analyzed by LA strain/strain rate in hypertensive and diabetic patients with high adiponectin. However, this relationship was not independent of other factors. We determined that age and hemoglobin were predictors of elevated adiponectin. Our study is the first to investigate adiponectin and LA mechanical functions using strain and strain rate analysis.

The present study showed that the association between adiponectin and AF might not be directly related to impaired LA mechanical functions; therefore, this relationship seems to be based on other mechanisms rather than LA fibrosis or myocyte loss. Macheret et al. found that adiponectin elevation was associated with incident $\mathrm{AF}$ independent of LA measurements and NT-pro BNP concentration [13]. Another study, included patients who underwent catheter ablation for AF, found similar results [14]. These studies showed that adiponectin elevation was associated with AF recurrence independent of LA volume and $\operatorname{LVEF}[1,14]$.
Although adiponectin is generally synthesized in adipocytes, it can also be synthesized in many other cell types such as bone, skeletal muscle, liver, and myocardium [15]. Local adiponectin synthesized in cardiomyocytes promotes glucose and fatty acid uptake and phosphorylation of 5 'adenosine monophosphate-activated protein kinase [16]. Ybarra et al. showed a paradoxical inverse relationship between LA volume and adiponectin in their study, including findings in obese patients [17]. These observations suggested that adiponectin might not cause atrial fibrosis directly but rather contributed to the interplay of other accompanying conditions responsible for the development of atrial fibrosis.

A disconnect between adiponectin and its receptor might be one of the underlying mechanisms responsible for our findings [18]. Prior studies demonstrated that adiponectin supplementation reduced the risk of myocardial fibrosis [19]. This supports adiponectin's antifibrotic role. Adiponectin also eliminates apoptotic cells and reduces inflammation. Thus, elevated adiponectin, which is considered to by anti-inflammatory and antifibrotic in patients with $\mathrm{AF}$, may be caused by disconnection between adiponectin and its receptor [19]. Increased gene expression of adiponectin secondary to receptor disconnection in the context of chronic inflammation may be the reason for elevated adiponectin [14]. Interestingly, we found that the LA strain rate was reduced in patients with elevated adiponectin, but other factors confounded this. This finding supported our adiponectin-receptor disconnection hypothesis.

Despite its beneficial effects, high adiponectin was associated with mortality and AF development in patients with CV diseases and heart failure [5]. The researchers hypothesized that this might be secondary to the dysfunction between adiponectin and its receptor [5]. It is assumed that chronic inflammation results in increased adiponectin production, and as the process persisted, adiponectin is unable to bind to its receptor and/or produces an insufficient post-receptor binding response within cells. Even if patients 
with elevated adiponectin had poorer LA mechanical function, this relationship was not independent of other factors supporting our hypothesis. Insufficient adiponectin in LA remodeling may cause LA fibrosis. Therefore, AF may develop in patients with high adiponectin. Further research at the molecular and cellular levels may clarify questions about this issue and perhaps pave the way for a new therapy for treatment of AF.

\section{Limitations}

The sample size of subjects was relatively small to generalize our findings. Because dedicated software for
LA strain had not yet been released, we used the current software for analysis of the LV to study LA strain.

\section{Conclusion}

In patients with $\mathrm{HT}$ and $\mathrm{DM}$, elevated adiponectin was associated with impaired LA mechanical function. Increased age and hemoglobin level were independent predictors of elevated adiponectin.

No conflict of interest is reported.

The article was received on 13/01/2021

\section{REFERENCES}

1. Yamaguchi N, Okumura Y, Watanabe I, Nagashima K, Takahashi K, Iso $\mathrm{K}$ et al. Clinical implications of serum adiponectin on progression of atrial fibrillation. Journal of Arrhythmia. 2017;33(6):608-12. DOI: 10.1016/j.joa.2017.07.009

2. Kizer JR, Barzilay JI, Kuller LH, Gottdiener JS. Adiponectin and Risk of Coronary Heart Disease in Older Men and Women. The Journal of Clinical Endocrinology \& Metabolism. 2008;93(9):3357-64. DOI: 10.1210/jc.2008-0640

3. Karas MG, Benkeser D, Arnold AM, Bartz TM, Djousse L, Mukamal KJ et al. Relations of Plasma Total and High-Molecular-Weight Adiponectin to New-Onset Heart Failure in Adults $\geq 65$ Years of Age (from the Cardiovascular Health Study). The American Journal of Cardiology. 2014;113(2):328-34. DOI: 10.1016/j.amjcard.2013.09.027

4. Kizer JR, Benkeser D, Arnold AM, Mukamal KJ, Ix JH, Zieman SJ et al. Associations of total and high-molecular-weight adiponectin with allcause and cardiovascular mortality in older persons: the Cardiovascular Health Study. Circulation. 2012;126(25):2951-61. DOI: 10.1161/ CIRCULATIONAHA.112.135202

5. Guo Y, Liu L, Wang J. Adiponectin and the risk of new-onset atrial fibrillation: a meta-analysis of prospective cohort studies. Bioscience Reports. 2019;39(6):BSR20182284. DOI: 10.1042/BSR20182284

6. Hao G, Li W, Guo R, Yang J-G, Wang Y, Tian Y et al. Serum total adiponectin level and the risk of cardiovascular disease in general population: A meta-analysis of 17 prospective studies. Atherosclerosis. 2013;228(1):29-35. DOI: $10.1016 /$ j.atherosclerosis.2013.02.018

7. Thomas L, Abhayaratna WP. Left Atrial Reverse Remodeling. JACC: Cardiovascular Imaging. 2017;10(1):65-77. DOI: 10.1016/j.jcmg.2016.11.003

8. Mondillo S, Cameli M, Caputo ML, Lisi M, Palmerini E, Padeletti $\mathrm{M}$ et al. Early detection of left atrial strain abnormalities by speckle-tracking in hypertensive and diabetic patients with normal left atrial size. Journal of the American Society of Echocardiography. 2011;24(8):898-908. DOI: 10.1016/j.echo.2011.04.014

9. Kawakami H, Ramkumar S, Nolan M, Wright L, Yang H, Negishi K et al. Left Atrial Mechanical Dispersion Assessed by Strain Echocardiography as an Independent Predictor of New-Onset Atrial Fibrillation: A Case-Control Study. Journal of the American Society of Echocardiography. 2019;32(10):1268-1276.e3. DOI: 10.1016/j. echo.2019.06.002
10. Psaty BM, Manolio TA, Kuller LH, Kronmal RA, Cushman M, Fried LP et al. Incidence of and Risk Factors for Atrial Fibrillation in Older Adults. Circulation. 1997;96(7):2455-61. DOI: 10.1161/01. CIR.96.7.2455

11. Imai E, Horio M, Nitta K, Yamagata K, Iseki K, Tsukamoto Y et al. Modification of the Modification of Diet in Renal Disease (MDRD) Study Equation for Japan. American Journal of Kidney Diseases. 2007;50(6):927-37. DOI: 10.1053/j.ajkd.2007.09.004

12. Gottdiener JS, Bednarz J, Devereux R, Gardin J, Klein A, Manning WJ et al. American Society of Echocardiography recommendations for use of echocardiography in clinical trials. Journal of the American Society of Echocardiography. 2004;17(10):1086-119. DOI: 10.1016/j. echo.2004.07.013

13. Macheret F, Bartz TM, Djousse L, Ix JH, Mukamal KJ, Zieman SJ et al. Higher circulating adiponectin levels are associated with increased risk of atrial fibrillation in older adults. Heart. 2015;101(17):1368-74. DOI: $10.1136 /$ heartjnl-2014-307015

14. Kim T-H, Lee JS, Uhm J-S, Joung B, Lee M-H, Pak H-N. High circulating adiponectin level is associated with poor clinical outcome after catheter ablation for paroxysmal atrial fibrillation. EP Europace. 2018;20(8):1287-93. DOI: 10.1093/europace/eux173

15. Piñeiro R, Iglesias MJ, Gallego R, Raghay K, Eiras S, Rubio J et al. Adiponectin is synthesized and secreted by human and murine cardiomyocytes. FEBS Letters. 2005;579(23):5163-9. DOI: 10.1016/j.febslet.2005.07.098

16. Gualillo O, González-Juanatey JR, Lago F. The Emerging Role of Adipokines as Mediators of Cardiovascular Function: Physiologic and Clinical Perspectives. Trends in Cardiovascular Medicine. 2007;17(8):275-83. DOI: 10.1016/j.tcm.2007.09.005

17. Ybarra J, Resmini E, Planas F, Navarro-López F, Webb S, Pou JM et al. Relationship Between Adiponectin and Left Atrium Size in Uncomplicated Obese Patients: Adiponectin, a Link Between Fat and Heart. Obesity Surgery. 2009;19(9):1324-32. DOI: 10.1007/s11695-009-9924-5

18. Shibata R, Izumiya Y, Sato K, Papanicolaou K, Kihara S, Colucci WS et al. Adiponectin protects against the development of systolic dysfunction following myocardial infarction. Journal of Molecular and Cellular Cardiology. 2007;42(6):1065-74. DOI: 10.1016/j. yjmcc.2007.03.808

19. Ouchi N, Walsh K. A Novel Role for Adiponectin in the Regulation of Inflammation. Arteriosclerosis, Thrombosis, and Vascular Biology. 2008;28(7):1219-21. DOI: 10.1161/ATVBAHA.108.165068 